\title{
La inversión acertada en la educación superior como desarrollo sostenible
}

\section{Successful investment in higher education as sustainable development}

\author{
Génesis Kristel Salazar Garcés
}

Escuela Superior Politécnica del Litoral, Ecuador

Lic. Norma Garcés Garcés. Mgs.

Lic. Alex Rene Villegas Bermeo

Universidad de Guayaquil, Ecuador

Autor para correspondencia: alex_villi84@hotmail.com / alexvillegasbermeo@gmail.com, norma.garces@hotmail.com, norma.garcesg@ug.edu.ec, genksala@espol.edu.ec

Fecha de recepción: 12 de Julio de 2016 - Fecha de aceptación: 30 de Agosto de 2016

Resumen: El desarrollo de la Educación sostenible es una propuesta nueva que intenta articular una serie de campos temáticos que se encuentran interrelacionados y que se están desarrollando de forma conjunta, es una oportunidad para encontrar y darle sentido a los procesos educativos mediante el desarrollo rural, derechos humanos, medio ambiente, genero, multiculturalidad y paz. La construcción de otro mundo posible, es una educación para la vida, que te permite prepararte para resolver problemas de la vida cotidiana, profesional y espiritual, y que está inmerso los derechos humanos, los problemas sociales, económicos y políticos, responde a los grandes problemas de la naturaleza. Los sistemas educativos actuales son muy verticales y están muy centrados en la competitividad sin solidaridad y son instrumentos de sustentabilidad. La gente y la sociedad deben empezar a reflexionar profundamente sobre la destrucción del planeta, educar y fortalecer una vida más sustentable y amigable con el entorno; es decir que se ha de reflexionar profundamente la forma de cómo se construye los sistemas educativos. Invertir en educación es lo más acertado en el presente y futuro porque impide que la pobreza se transmita de generación en generación y te ofrece mejores oportunidades de empleo.

Palabras Claves: desarrollo; sostenible; innovación; educación e inversión

\begin{abstract}
The development of education is a new proposal that seeks to articulate a number of thematic areas that are interrelated and are developing jointly, is an opportunity to find and make sense of educational processes through the environment, rights human, gender, rural development, multiculturalism and peace. The construction of another possible world, is an education for life that allows you to prepare to solve problems of everyday, professional and spiritual life, and is immersed human rights, social, economic and political, responds to the great nature problems. Current education systems are vertical and are very focused on without solidarity and competitiveness are instruments of unsustainability. People and society must begin to reflect deeply on the destruction of the planet, educate and build a more sustainable and environment friendly life; it must reflect deeply how education systems is built. Investing in education is the most successful in the present and future because it prevents poverty is transmitted from generation to generation and offers better employment opportunities.
\end{abstract}

Key words: development sustainable; innovation; superior education and investment 


\section{Introducción}

El Ecuador contará con un nuevo plan decenal de educación que es un instrumento de gestión estratégica planteado para implementar un conjunto de acciones pedagógicas, técnicas, administrativas y financieras, que oriente los procesos de modernización en los sistemas educativos. La finalidad de este sistema es establecer grandes objetivos entorno a la educación, a la aplicación de estándares de calidad, cobertura y rectoría-administración.

La propuesta de este plan que se inició en agosto del 2015, es un accionar que se ajusta a la realidad y entorno a nuestro país, para tener un país más justo, equitativo, que brinde oportunidades a todas las personas. En el plan nacional decenal contempla ocho políticas.

- La Universalización de la Educación Inicial.

- La Universalización de la Educación General Básica.

- El incremento del Bachillerato.

- Reducción del Analfabetismo.

- Mejoramiento para la infraestructura y el equipamiento educativo.

- Mejoramiento de la calidad de la Educación

- Revalorización de la profesión Docente.

- Incremento del presupuesto en la Educación.

En el tema de la calidad de la educación se pretende garantizar oportunidades de aprendizaje, para desarrollar una comunidad educativa, justa, solidaria e innovadora. El desarrollo sostenible que se observa en la educación como una forma de cubrir de forma adecuada las necesidades humanas, pero sin transgredir los límites ecológicos del planeta. El respeto por los diversos sistemas de conocimiento y cultura sirve de base para un desarrollo sostenible y es por ello que la educación formal y no formal, benefició el desarrollo y crecimiento económico.

Según UNESCO(2015): La educación es un derecho fundamental y la base del progreso de cualquier país. Los padres necesitan tener conocimientos sobre salud y nutrición para poder brindar a sus hijos la infancia que se merecen. Para ser prósperos, los países necesitan trabajadores cualificados y educados. Los desafíos de la erradicación de la pobreza, la lucha contra el cambio climático y el logro de un desarrollo verdaderamente sostenible en los próximos decenios nos conminan a actuar juntos. Con colaboración, liderazgo e inversiones acertadas en educación se puede transformar la vida de las personas, las economías de los países y nuestro mundo en general (pág. 1).

En todas las etapas de la vida la educación ha sido siempre transcendental en el desarrollo de un país, con visión para cumplir sus metas y objetivos. Esto implica la evolución económica, social, cultural, política, tecnológica y educativa dentro de la sociedad. La sostenibilidad de una educación permite satisfacer necesidades de generaciones presentes y futuras, consiente de cuidar el medio ambiente natural.

Según Aarón Benavot, director del Informe de Seguimiento de la Educación para todo en el Mundo “... aporta pruebas contundentes sobre la interacción dinámica entre la educación y 
otras prioridades del desarrollo, y recuerda que solo actuando juntos se puede alcanzar un desarrollo integral, equitativo y duradero" (UNESCOPRESS, 2014, pág. 3).

Una de las estrategias educativas que ayude a mejorar la educación superior es modificar dentro de las mallas curriculares contenidos que sustente el desarrollo razonable y sustentable, con la inclusión de asignaturas de educación en valores, ética profesional, derechos universales, equidad, inclusión y promover la educación democrática ciudadana, el respeto a una sociedad justa.

La educación se sustenta en un nuevo paradigma educativo que responda a transformaciones sociales, económicas, políticas, productivas, y que está inmerso al desarrollo del conocimiento. Una sociedad sin reglas, sin normas, sin respeto hacia los demás no conduce a nada; en cambio la aplicación de políticas acertadas, conlleva al buen uso del poder público para conseguir una ventaja.

En la actualidad, las causas principales del atraso en la educación es una gestión pública educativa de procesos lentos para ejecutar acciones y planes que beneficien una educación dinámica, la toma de decisiones en asuntos prioritarios para una mejora necesaria, conducta de funcionarios públicos que cometen actos ilícitos, enriquecimiento ilegal, que perjudica el resultado de un buen manejo del recurso del país.

Desde el año 2012, se ha visto cambios paulatinos dentro de la educación pública y estos pasos que se están dando deben consistir en inculcar la teoría, la praxis, los fundamentos sostenibles de una protección para el medio ambiente, los requerimientos adecuados, establece (OIUDSMA, 2014), que su principal compromiso consiste en el replanteamiento de las funciones esenciales de la Educación Superior: la docencia, la investigación, la extensión y la gestión con el objetivo de configurar una nueva cultura institucional, que se orienta por los principios del desarrollo sostenible, de tal forma que permita concienciar y responsabilizar a toda la comunidad universitaria la necesidad de actuar de acuerdo con unas prácticas ambientalmente coherentes, como con los principios de la solidaridad con los pueblos del mundo y con las generaciones futuras (pág. 3).

Este trabajo se estudia en dos principales propuestas que se deben alcanzar:

\section{El plan decenal de educación}

Plan decenal de educación 2016-2025 plantea algunos logros que se deben de cumplir como son la cobertura en infraestructura, personal docente calificado a nivel nacional y el espacio de aprendizaje máximo 25 alumnos por aula, contratar personal docente con vocación y con alta valoración social, establecer que todas las unidades educativas cuenten con infraestructura y equipamiento de calidad; actualizar el currículo basado en la realidad de nuestro entorno y los estándares de aprendizajes.

\section{Las metas a nivel macro y micro}


La innovación y la educación online, el plan de progresión estudiantil, donde se establecerán cámaras afuera de las instituciones educativas, que cuenten con más de mil alumnos, departamento de consejería en bienestar estudiantil.

El ministerio de Educación detalló, el objetivo para el 2018 es que se ubique nuestro país, como uno de los mejores sistemas educativos en América Latina y en el mundo y que los principales autores de este proceso son los maestros, estudiantes, directivos y padres de familia, con respecto a otras preocupaciones, se enfatiza que pondrán en ejecución un plan de gestión de seguridad para los estudiantes, tomando consideraciones hacia los problemáticas actuales en los adolescentes como el consumo de drogas, alcohol, tabaco.

La prevención y disminución de embarazos adolescentes y el acoso escolar son temas que se planea realizar acciones de cambios positivos para beneficios de los jóvenes.

\begin{tabular}{lr}
\hline \multicolumn{2}{c}{ Inversión Publica Social } \\
\hline Entidad & Proforma 2016 \\
& \\
Ministerio De Educación & $703^{\prime} 070.677,00$ \\
Ministerio De Inclusión Económica Y Social & $37^{\prime} 960.568,09$ \\
Total & $741^{\prime} 031.245,09$ \\
\hline
\end{tabular}

La inversión en Educación durante el periodo 2007-2015 ha sido de 11.746000.000,00.

La educación que se desea alcanzar es mejorar la calidad y la equidad, para erradicar la pobreza y la desigualdad que aún existe en nuestro entorno educativo, la decisión tomada es abordar retos que aún no están resueltos, como el abandono escolar, el bajo rendimiento de los alumnos y el desinterés de los alumnos por adquirir los nuevos conocimientos. Se ha desarrollado nuevos modelos de enseñanza y en el aprendizaje con la incorporación de las TIC, apuesta por la creatividad, innovación y el desarrollo de la investigación tanto filosófica como científica. Es fundamental el desarrollo necesario de todas las mejoras en el sistema educativo.

La presentación de esta investigación sirve para reforzar el posicionamiento de la educación ecuatoriana con los diferentes países, que se adapten proyectos específicos en la que exista la vinculación con la sociedad, compromiso solidario del estado, con mayores recursos para contribuir las metas propuestas, la formación de los docentes, la creación de asociaciones de padres de familias, instituciones universitarias, empresas, organizaciones sociales, contribuirá a formar alianzas estratégicas y que participen organizaciones nacionales e internacionales de fuentes de financiación que ayuden a las naciones y los países con mayores dificultades educativas, que conlleva a la organización de sociedades democráticas equilibradas y justas.

El alcance de todos los cambios planteados en esta investigación, tendrá como consecuencia una educación estable y toma de la conciencia. La preocupación por el medio ambiente, un cambio de pensamiento y el compromiso de todos los ciudadanos especialmente de las personas que tienen un mayor nivel de educación, según el análisis de estudio en Global Warming Citizen Survey, se observó que si el nivel de educación de las personas eran mayor aporta calidad de la educación y es crucial para el desarrollo económico de cada individuo. 
El énfasis que aporta a esta investigación se respalda lo planteado por (BOKOVA, 2015) Los beneficios de la educación impregnan todos los ámbitos de la vida desde el momento mismo del nacimiento. Si se quiere erradicar la pobreza y el hambre, mejorar la salud, proteger nuestro planeta y construir unas sociedades más incluyentes, resilientes y pacíficas, se debe brindar a toda persona el acceso a una educación de calidad a lo largo de toda la vida, poniendo especial atención en las oportunidades de las niñas y las mujeres. Las pruebas son indiscutibles, la educación salva vidas y transforma vidas; es el fundamento de la sostenibilidad. Por ello, debe haber una colaboración entre todos los sectores del desarrollo para que la educación sea un derecho universal. (pág. 24)

Existen muchos respaldos sobre la garantía educacional, tratados, pactos y acuerdos, que tienen una finalidad, el logro del desarrollo sostenible en la educación, pero en el Ecuador ya existe partes interesadas trabajando conjuntamente que se han comprometido de una transformación en la educación.

Puede verse un paso importante en el lineamiento de los cambios educacionales; en este período educacional, existe un máximo interés en el desarrollo profesional de parte de la mayoría, entendiendo lo que significa la educación en su vida, ofrecer mejores medios de vida, impide que la pobreza se transmita de una generación a otra, la educación se convierte en un pasaporte para ser parte de la población activa, puede reducir la desigualdad, la conservación y protección del medio ambiente, y muchos beneficios existentes que se alcanzan con mayor preparación.

\section{Materiales y Métodos}

El diseño de este trabajo es una investigación cualitativa, analítica y descriptica en el que se describe la temática de lo que acontece y los cambios que se han logrado en cuanto a la educación superior con propósitos y principios como los derechos universales, la preservación del planeta, la erradicación de la pobreza, creación de un desarrollo económico sostenido, inclusivo y sostenible e inclusión social.

\section{Resultados y discusión}

Las realidades del Ecuador son muy diferentes de otros países del mundo y el nivel de desarrollo depende del crecimiento económico con que progresa el país, para que la educación sea sostenible se ha comprobado que las formas de progresar es una educación de calidad, salud, bienestar físico, psicológico, mental y social, el derecho al agua potable y el saneamiento.

La investigación obtenida ha permitido conocer los criterios necesarios para realizar una propuesta que promueva mejorar la gestión institucional, los procesos en la educación y contar con material tecnológico que ayude en el proceso de enseñanza-aprendizaje y que sea una prioridad ineludible.

Si toda persona tiene derecho a una educación de calidad, el ingreso per capital (El ingreso hace referencia a todas las entradas económicas que recibe una persona, una familia, una 
empresa, una organización, etc.), podría aumentar las ventajas económicas en su familia, crece el aumento en el gasto público y por ende el desarrollo y progreso en un país.

\section{Conclusiones}

La propuesta es realizar un plan de acción que beneficie a todas las personas, erradicar la pobreza en el Ecuador, y para ello se requiere un pueblo educado con amplia libertad para un desarrollo sostenible.

Liberar a toda la humanidad de la miseria, privaciones e implementar un plan de salud que ayude a los más necesitados y adquirir el compromiso de realizar grandes transformaciones para conducir al planeta hacia la sostenibilidad del planeta en lo económico, social y político.

Es una decisión importante de poner fin a la pobreza y al hambre, inculcar que los seres humanos puedan disfrutar con tranquilidad, igualdad y dignidad en un ambiente armónico y saludable.

La alianza Mundial para el desarrollo sostenible se basa en un espíritu de solidaridad y en la colaboración de todos los países, pero para cumplir esta meta el ser humano debe despojarse de tantos pecados como la envidia.

La paz, la prosperidad, el cuidado del planeta son referentes de gran realce para que se cumpla los objetivos planteados.

\section{Bibliografía}

BOKOVA, I. (2015). El desarrollo sostenible comienza por la educacion. En Unesco, El desarrollo sostenible comienza por la educacion (pág. 24). UNESCO/Karel Prinsloo/ARETE.

Ministerio de Educacion (2016). Comunidad educativa participa en socialización de evaluación docente y ejes principales de la propuesta del Plan Decenal de Educación. Retreive from http://educacion.gob.ec/comunidad-educativa-participa-en-socializacion-evaluaciondocente-y-ejes-principales-de-la-propuesta-del-plan-decenal-de-educacion/

OIUDSMA. (2014). La Organización Internacional de Universidades por el Desarrollo Sostenible y el Medio Ambiente. La Educación Superior Para El Desarrollo, 3.

Ramírez Sánchez, R. (2015) La Educación Superior Para El Desarrollo Sostenible. XII Coloquio Internacional de Gestion Universitaria.

Segovia Baus, F. (Agosto 2016) Apuntes para el nuevo Plan Decenal de la Educación 2016-2025. EL COMERCIO. Retrieve from http://www.elcomercio.com/blogs/la-silla-vacia/apuntesnuevo-plan-decenal-educacion.html. Si está pensando en hacer uso del mismo, por favor, cite la fuente y haga un enlace hacia la nota original de donde usted ha tomado este contenido. ElComercio.com 
UNESCO. (15 de agosto de 2015). http://wwwefareport.unesco.org. Recuperado el 20 de junio de 2016, de http://wwwefareport.unesco.org: http://es.unesco.org/gem-report/sites/gemreport/files/UNGA_PR_sp.pdf

UNESCOPRESS. (08 de 09 de 2014). http://www.unesco.org. Recuperado el 18 de 06 de 2016, de http://www.unesco.org: http://www.unesco.org/new/es/media-services/singleview/news/unesco_sustainable_development_begins_with_education/\#.V2yTstLhCM8 